\title{
¿Estaba intoxicado por cocaína este individuo? (I): estimaciones basadas en la farmacocinética de la droga.
}

\author{
Was this individual poisoned by cocaine? (I): estimations \\ based on the pharmacokinetic of the drug.
}

\section{A. Ortega Pérez ${ }^{1}$}

\section{RESUMEN}

La cocaína tiene mucho interés para los médicos forenses a causa de la frecuencia de su uso, de su toxicidad y de su implicación en hechos violentos. Con el fin de ayudar a dictaminar si existió una intoxicación por la droga se exponen los datos disponibles sobre su farmacocinética.

La droga puede absorberse por varias vías, alcanzando concentraciones sanguíneas apreciables casi inmediatamente y las concentraciones máximas en pocos minutos o en una hora, según la vía. La cocaína tiene una semivida en la sangre de entre 40 y 90 minutos, según los estudios, de forma que desaparece de esta en pocas horas. Sin embargo, puede detectarse en la orina durante $\mathbf{2 4}$ horas o más. Las correlaciones entre cocaína sanguínea y cocaína cerebral o biliar pueden ayudar a estimar la dosis tomada y el tiempo transcurrido desde la administración.

Según los datos disponibles, las concentraciones de cocaína halladas al analizar la sangre o los tejidos son tan variadas que por sí mismas no son concluyentes para determinar si una persona estaba intoxicada o no. En el artículo se aportan algunas sugerencias para aumentar los datos disponibles y para mejorar la estimación y la calidad de la peritación.

Palabras clave: Aspiración, cocaína, farmacocinética, inhalación, inyección, peritación, semi-vida, variaciones.

\section{ABSTRACT}

Cocaine is of considerable interest to forensic doctors, because it is widely used, is very toxic and is frequently implicated in acts of violence. To help the expert witness decide on cocaine intoxication the available pharmacokinetic data are summarized here.

The drug can be absorbed through different routes. Measurable blood concentrations are reached almost immediately with maximum concentrations within minutes, or within an hour depending on the route of administration. Depending on the study from which the data are drawn, the half-life of cocaine appears to be between 40 and 90 minutes.

The substance disappears from the blood over several hours, but can be detected in urine for 24 hours or more. The correlations between blood cocaine concentrations and those in the brain or bile can help estimate the dose absorbed and the time elapsed since its intake.

In short, current data suggest that the concentrations of cocaine observed are so variable that by themselves no definite conclusions can be drawn regarding an individual's intoxication based merely on the concentration of cocaine noted in one sample. In this present communication some suggestions are provided for increasing the available data and for improving the estimation as well as the quality of the clinical judgement.

Key words: Aspiration, cocaine, expert witness, halflife, inhalation, injection, pharmacokinetics, variations.

Correspondencia: Dr. Arturo Ortega. Unidad de Medicina Legal. Facultad de Medicina y Ciencias de la Salud. C/ Sant Llorenç, 2143201 - Reus (Tarragona). Correo electrónico: aop@fmcs.urv.es

\footnotetext{
1 Doctor en Medicina. Profesor de Medicina Legal y Toxicología. Facultad de Medicina y Ciencias de la Salud. Universidad "Rovira i Virgili".
} 


\section{IMPORTANCIA DE LA COCAÍNA PARA EL MÉDICO FORENSE}

En los años ochenta se inició una epidemia de uso de cocaína que la convirtió en un problema de salud pública en el mundo. Su uso no ha remitido y se estima que incluso aumentará. El $1 \%$ de los adultos estadounidenses y el 2,5 \% de los adolescentes jóvenes han tomado cocaína en el último mes [I].

La cocaína tiene interés para los médicos forenses porque es tóxica, tanto al tomarla sola como asociada a otras drogas o a fármacos legales. Asimismo, el uso de cocaína a menudo se asocia a hechos violentos, con traumatismos accidentales o voluntarios.

Por ejemplo, en la ciudad de Nueva York murieron a causa de un traumatismo | 4.843 personas entre 1990 y 1992. Al analizar la sangre y la orina de estas, hallaron benzoilecgonina (uno de los metabolitos de la droga) en el 27 \% de los fallecidos y cocaína libre en el $18 \%[2]$.

Aproximadamente un tercio de las muertes se debió a intoxicación por droga, pero dos tercios los causaron traumatismos derivados de homicidios, suicidios, accidentes de tráfico y caídas. Entre los ciudadanos de Nueva York la muerte tras el consumo de cocaína estaba entre las cinco primeras causas de muerte en las personas de entre 15 y 44 años.

En una investigación realizada en el estado de Georgia (Estados Unidos) más del 30 \% de las víctimas de homicidio tenían benzoilecgonina en la sangre [3]. El porcentaje era aún mayor entre los fallecidos por arma de fuego. También se ha implicado a la cocaína en el fallecimiento durante el juego de la ruleta rusa, porque dos tercios de los fallecidos en la ciudad de Nueva York durante la práctica de ese juego la habían tomado poco antes [4].

Con respecto a España, del Río y cols. [5], recogen en un artículo muy útil los análisis realizados en el Instituto Nacional de Toxicología en busca de sustancias psicoactivas (alcohol, drogas o psicofármacos) en los 59 07 I conductores fallecidos en accidentes de tráfico en el país entre | 99 | y 2000. En casi el $10 \%$ de esos fallecidos, concretamente en 5745 , se tomaron muestras de sangre (los porcentajes han ido en aumento, desde menos del $5 \%$ en 199। hasta el $25 \%$ en 2000). En el $50 \%$ de las muestras analizadas se detectó alguna sustancia psicoactiva, siendo el alcohol la más común (en el 44 \% de las muestras), seguida por las drogas ilegales (9 \%) y por los psicofármacos (5\%). Con frecuencia se halló más de una sustancia psicoactiva en la sangre de los fallecidos.

Por lo que se refiere específicamente a la cocaína, en 300 de estas muestras (el 5,2 \%) se halló benzoilecgonina. En la mayoría de los casos (246 muestras, el 80 \%) había asociadas otras sustancias psicoactivas: alcohol ( 63 casos, el $54 \%$ de las combinaciones), otras drogas ilegales ( I 19 , el $40 \%$ ) o medicamentos (52 casos, el $17 \%$ ).

Todos estos son los resultados de los análisis de laboratorio, pero el juez pide a los médicos forenses interpretarlos e informarle sobre la influencia que la sustancia psicoactiva pudo ejercer sobre el individuo. Esta revisión de la farmacocinética de la cocaína quiere proporcionar datos que ayuden a dicha interpretación: la farmacocinética de la droga, las distintas muestras utilizables para determinarla y sus resultados, tanto en la persona viva como en el cadáver.

\section{FARMACOCINÉTICA DE LA COCAÍNA}

La administración de cocaína induce unas cocainemias primero crecientes, hasta un máximo o pico, y después decrecientes, a medida que se elimina la droga. Es una gráfica en " $V$ invertida", pero de ramas muy desiguales, cuyo esquema se presenta en la Figura I. 


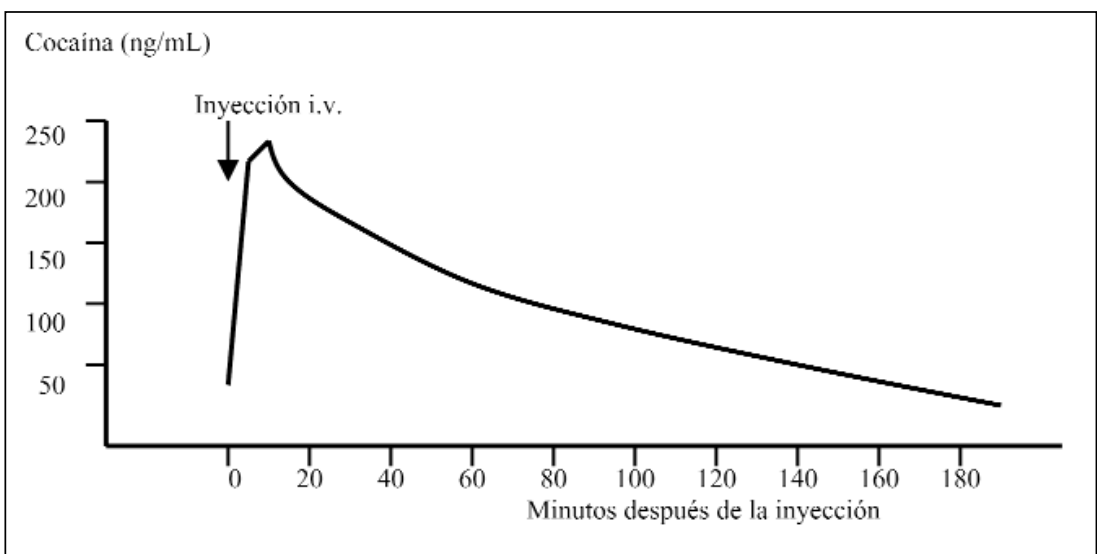

Figura 1. Concentración sanguínea de cocaína en función del tiempo transcurrido desde su administración. Adaptado de Halpern y cols. [10].

(No se especifican las unidades de tiempo porque la gráfica se refiere a varios tipos de muestras).

\section{A. ABSORCIÓN DE LA COCAÍNA Y MÁXIMOS PLASMÁTICOS.}

La cocaína puede absorberse tras administrarla por diferentes vías: aspiración ("esnifado"), inhalación (fumando la cocaína base), inyección intravenosa o ingestión.

\section{- Cocaína aspirada.}

Una "raya" de clorhidrato de cocaína contiene entre 10 y 35 mg de la droga, según su pureza [6]. La cocaína aspirada se absorbe muy rápidamente y lleva a máximos plasmáticos a los I560 minutos $[6,7]$.

Después de aspirar una dosis de $1,5 \mathrm{mg} / \mathrm{kg}$ de cocaína se alcanza una concentración plasmática máxima en un abanico entre los 120 y los 474 ng/mL [6]. Una dosis algo mayor, de 2 $\mathrm{mg} / \mathrm{kg}$, llevó a un pico plasmático promedio de cocaína en el abanico anterior, de $161 \mathrm{ng} / \mathrm{mL}$ una hora después [6].

La cocaína también puede administrarse sobre las mucosas oral o genital. La administración oral de 2 mg/kg de cocaína lleva a picos plasmáticos a los 50-90 minutos de la administración y de magnitud similar a los conseguidos por la vía intranasal [6].

\section{- Cocaína inhalada.}

Se inhalan los productos de la combustión del hidrocloruro de cocaína o de la cocaína base (crack). La cocaína inhalada pasa inmediatamente a la sangre, como mínimo tan rápido como tras la inyección, porque la mayoría de ella llega a los pulmones en las primeras cuatro aspiraciones del cigarrillo [6]. Evans y cols. [8] investigaron las cocainemias de nueve voluntarios que fumaron entre 12,5 y 50 mg de cocaína. Alcanzaron sus máximas concentraciones arteriales en los primeros I5 segundos tras la inhalación y las venosas, casi 4 minutos después. Las cocainemias arteriales fueron bastante mayores que las venosas.

En la investigación de Jenkins y cols. [9] llegar a los picos plasmáticos tardó un poco más: 2 minutos después de fumar dosis de 20 o de 40 mg y 5 minutos tras una dosis menor, de $10 \mathrm{mg}$.

\section{- Cocaína intravenosa.}

La concentración máxima de cocaína en la sangre se alcanza 4-6 minutos después de inyectarla, aun- 
que según los autores, puede tardar hasta 8 minutos [ [0]. Son intervalos similares a los necesarios para alcanzar la concentración máxima de cocaína en el cerebro.

\section{- Cocaína oral.} ingerirla $[\mathrm{ll}]$.

La concentración máxima de cocaína en la sangre se alcanza unos 60 minutos después de

\section{B. ELIMINACIÓN DE LA COCAÍNA Y SEMIVIDA EN SANGRE.}

La cocaína desaparece con rapidez de la sangre y de los órganos. La expresión matemática de esta velocidad de desaparición es su semivida, es decir el tiempo que tarda la concentración de esta en reducirse a la mitad. Los distintos investigadores citan semividas diferentes para la cocaína: unos 40 minutos [12]; 48 minutos [13]; 65 minutos [1 4], 69 minutos [1 5] o hasta 90 minutos $[2,16]$.

Este abanico tan amplio se debe al tamaño de los grupos experimentales, de seis a diez individuos en muchos casos, y también a la variabilidad interpersonal en la eliminación de la droga [12].

La cocaína se transforma en varios metabolitos distintos, de los cuales la benzoilecgonina es el mayoritario. Cada individuo tiene su velocidad de eliminación de cocaína, determinada en buena parte por la concentración o la actividad de sus colinesterasas. Estos enzimas participan en dos vías de degradación de la cocaína: a metiléster de ecgonina y a benzoilecgonina. Esta última biotransformación es a la vez enzimática, catalizada por esterasas, y no enzimática, es decir espontánea. La catálisis mediante esterasas es el mecanismo predominante en la persona viva [18].

De la misma forma, los usuarios crónicos eliminan más lentamente la cocaína que los usuarios esporádicos u ocasionales, aunque de nuevo las estimaciones sobre su semivida sanguínea varían. Jufer y cols. investigaron los efectos de la administración oral crónica de cocaína a seis personas sanas [ 16] y distinguieron dos fases distintas en la eliminación: Durante la administración aguda, la semivida de la cocaína en plasma fue de I,5 $\pm 0,1$ horas. En la segunda fase, que llamaron terminal, la semivida sanguínea fue de $19 \pm 4,2$ horas (este parámetro sólo pudieron determinarlo en tres de los seis sujetos, debido a la interferencia analítica causada por las altas concentraciones de benzoilecgonina). En cambio, Moolchan y cols. [18], calcularon una semivida plasmática mucho más corta para la cocaína durante la fase terminal, de sólo 3,8 horas.

\section{Muestras para la determinación de cocaína.}

Para muertes relacionadas con drogas y fármacos, Drummer y Gerostamoulos recomiendan, en su revisión de 2002 [19], recoger muestras de sangre, de orina, de humor vítreo, de contenido gástrico, de bilis y de hígado. En la persona viva pueden tomarse también muestras de saliva, de sudor o de cabello [20].

Dada la sensibilidad de las técnicas de detección actuales, se encontrarán cocaína y sus metabolitos en cualquier órgano analizado de la persona que la tomó.

\section{- Cocaína en sangre.}

Drummer y Gerostamoulos recomiendan [19], usar sangre de la vena femoral para los análisis toxicológicos, siempre que sea posible, porque es la que parece rendir una concentración de tóxico más similar a la existente en el momento de la muerte. En cambio no tienen a la sangre cardíaca, en general, por una muestra apropiada para el análisis cuantitativo, ya que la concentración de drogas y fármacos en ella es casi invariablemente más alta que en la sangre femoral. 
Sin embargo, Vanbinst y cols. [2 I] no apoyan esa recomendación, tras analizar las concentraciones en sangres cardíaca y femoral y en bilis de distintas sustancias químicas. No hallaron diferencias significativas en las concentraciones de cocaína entre las sangres de los distintos puntos.

\section{- Cocaína urinaria.}

Tras aspirar una dosis de 1,5 mg de cocaína/kg de peso, la cocainuria máxima se halló en las primeras dos horas y después disminuyó rápidamente, aunque continuó siendo detectable durante las 24 horas siguientes a la aspiración [22]. A partir de esos datos, en los libros de referencia se indicaba que la incapacidad para detectar la droga en orina sugería un lapso mayor de 10 horas desde la administración de esta [6]. Hoy día esa aseveración ha quedado obsoleta, dada la capacidad de detección real, mucho mayor. Sin embargo, continúa siendo cierto que durante unas horas puede hallarse cocaína en la orina a pesar de que haya desaparecido de la sangre.

Jufer y cols. dieron una semivida de 4,I $\pm 0,9$ horas para la fase aguda de la eliminación de la cocaína urinaria cuando investigaron los efectos de la administración oral crónica de cocaína a seis personas sanas [16].

\section{- Cocaína en el cerebro.}

La cocaína difunde con facilidad hacia el cerebro y en el momento del pico plasmático la concentración cerebral es aproximadamente cuádruple de la sanguínea [6]. La concentración de aquella disminuye más lentamente que la de esta, un hecho que puede ayudar a estimar el tiempo transcurrido desde la administración. El cociente cocaína cerebral / cocaína sanguínea en las muertes que ocurren entre I y 3 horas tras la administración es de una media de 9,6 [23] y puede llegar a 20 [6]. En los casos en los que la toma de cocaína no tuvo que ver con la causa de la muerte, el cociente cerebro / sangre tuvo una media de 2,5 [23].

\section{- Cocaína en la bilis.}

La bilis se ha usado poco como muestra para detectar cocaína y/o sus metabolitos. Sin embargo, Vanbinst y cols. la consideran muy útil, dado que en ella los fármacos, las drogas y sus metabolitos se hallan por lo general a concentraciones superiores a las de la sangre [2 I]. Incluso en más de un tercio de los casos no detectaron la sustancia en la sangre y sí en la bilis. Por eso es útil enviar una muestra de bilis para su análisis, que complemente los resultados de los otros líquidos o matrices biológicas, particularmente cuando la persona ha sobrevivido durante varios días después de la toma de cocaína.

\section{- Cocaína en el humor vítreo.}

Mackey-Bojack y cols. [24] analizaron muestras de 62 cadáveres para estudiar la utilidad del humor vítreo como espécimen para cuantificar la cocaína y sus metabolitos. Las concentraciones de cocaína en humor vítreo y en sangre fueron similares, dentro de su variabilidad: 0,61 $\pm 0,99 \mathrm{mg} / \mathrm{L}$ en la primera muestra y 0,49 $\pm 1,20$ en la segunda.

McKinney y cols. estudiaron los cambios postmortales de la concentración de la cocaína en cerdos [25]. Les inyectaron cocaína intravenosa y cinco minutos después los mataron. En ese momento y de nuevo ocho horas después tomaron muestras de sangre de la vena femoral y del humor vítreo. Aunque no aumentaron significativamente con el tiempo las concentraciones medias de cocaína, sí existió una gran variabilidad en la dirección y en la magnitud de los cambios en cada animal. 


\section{INTERPRETACIÓN DE LA CONCENTRACIÓN DE COCAÍNA EN UNA MUESTRA}

Teniendo en cuenta las consideraciones farmacocinéticas, se comprende que la concentración de cocaína en una muestra dependerá de varios parámetros, en especial de la dosis administrada y del tiempo transcurrido desde entonces (Figura 2). Por eso en la práctica los resultados de los análisis dan unas cifras muy variadas, en función de la situación concreta.

Así por ejemplo Williams et al. [26] determinaron las concentraciones de cocaína en plasma y en orina de 29 pacientes que habían acudido al Servicio de urgencias y a quienes habían dado positivos los cribados urinarios de benzoilecgonina. Compararon esos resultados con el diagnóstico de entrada y con la historia clínica. Sólo hallaron cocaína en el plasma de tres personas (a concentraciones entre 16 y $130 \mathrm{ng} / \mathrm{mL}$ ) aunque la había en la orina de 23 (pero a concentraciones de entre 4 y $40 \mathrm{ng} / \mathrm{mL}$ ). No encontraron ninguna correlación entre la historia clínica del paciente, su diagnóstico de entrada o sus síntomas frente a las concentraciones plasmáticas o urinarias de cocaína o de alguno de sus metabolitos.

Algo similar les ocurrió a

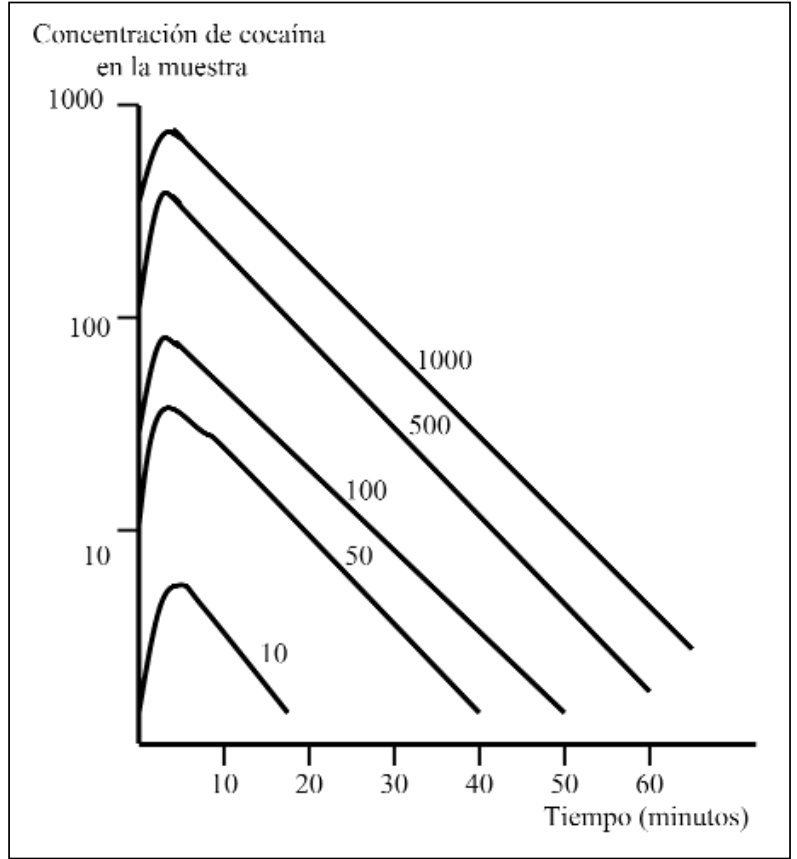

Figura 2. Relacion esquemática entre las concentraciones de cocaina en una muestra determinada, la dosis administrada de esta y el tiempo transcurrido desde la toma.

Shimomura y cols. [27], quienes determinaron la concentración de cocaína en varias muestras de 15 personas en cuyo fallecimiento estaba implicada la droga. Obtuvieron variaciones de varios órdenes de magnitud entre los individuos. Expresando las concentraciones como mediana (amplitud), los resultados fueron: en el hígado, $57 \mathrm{ng} / \mathrm{g}$ (de 0 a 503); en el cerebro, $187 \mathrm{ng} / \mathrm{g}$ (de 0 a | 403); en la sangre, $12 \mu \mathrm{g} / \mathrm{L}$ (de 0 a 88) y en la orina, $1208 \mu \mathrm{g} / \mathrm{L}$ (de 37 a 28 062).

Jenkins y Goldberger [28] analizaron la sangre cardíaca de trece fallecidos por causas relacionadas directamente o no con la cocaína. Las cocainemias halladas variaron entre 23 y $2088 \mathrm{ng} / \mathrm{mL}$.

Karch y cols., por su parte, [29] compararon las cocainemias de 48 hombres fallecidos a causa del uso de cocaína con las de 51 hombres, usuarios de la droga pero que murieron por un traumatismo. Las cocainemias de unos y otros no fueron significativamente distintas: I, I 2 frente a 0,49 $\mathrm{mg} / \mathrm{L}(p=0,10)$. Sin embargo, recogieron otros datos que pueden ayudar a discriminar el papel de la cocaína: los fallecidos a causa de esta eran más delgados (IMC de 24,6 frente a 30,6, p < 0,000 I), pero tenían corazones, pulmones, hígados y bazos más pesados (426 g frente a 369, $p=0,009$; 1275 g frente a I007, $p=0,009$; 1896 g frente a $1628, p=0,008$ y $193 \mathrm{~g}$ frente a $|46, p=0,00|$, respectivamente). 
A la variabilidad en la persona viva hemos de añadirle la posibilidad de redistribución post-mortal de la cocaína, que sería otro elemento de incertidumbre para la peritación sobre el papel de la cocaína en una muerte, a partir de una cifra del laboratorio. La redistribución existe, pero no está claro en qué muestras son más altas las concentraciones y en cuáles más bajas ni en qué sentido cambian estas ni tampoco la magnitud de los cambios.

Logan y cols. estudiaron la cuestión [30] en fallecidos en relación con la droga cuantificando la cocaína en dos ocasiones: poco tiempo tras la muerte y durante la autopsia. Recogieron muestras de varios puntos: sangre de la vena femoral, del ventrículo cardíaco y del líquido cefalorraquídeo. Las concentraciones de cocaína variaron sin seguir patrones consistentes según el lugar de la toma, ni tampoco fueron coherentes la dirección o la magnitud del cambio de concentración con el paso de las horas.

Lo mismo les ocurrió a Hearn y cols. [3 I], quienes investigaron si las cocainemias variaban en las primeras horas tras la muerte. Tomaron muestras en dos momentos: la primera tan pronto después de la muerte como les fue posible y la segunda durante la autopsia. Generalmente, pero no siempre, la cocainemia en la vena subclavia disminuyó durante esas horas, mientras que aumentaron las cocainemias cardíaca, aórtica y de la vena femoral.

Los investigadores citados [29,30,3 I ] concluían que las concentraciones postmortales de cocaína no reflejan necesariamente las que hubieron en el momento de la muerte y no deberían ser el elemento principal para determinar la causa de muerte cuando se sospeche la intervención de la cocaína [30]. Es decir, que las cocainemias postmortales por sí mismas no permiten evaluar o predecir la toxicidad causada por la droga.

\section{SUGERENCIAS PARA MEJORAR LAS ESTIMACIONES DE LOS PERITOS}

Hoy por hoy los resultados analíticos por sí mismos no son concluyentes. Las concentraciones son demasiado variables y falta una correlación con la implicación causal de la droga en la muerte. Por eso se necesitan más datos sobre las muertes en las que está presuntamente implicada la cocaína, que permitan llegar a conclusiones de interés para la justicia. Para obtenerlos sería aconsejable:

a) Realizar más ensayos clínicos sobre la farmacocinética de la cocaína, en los que se administren varias dosis de droga y se recojan varias muestras en diferentes momentos.

b) Recoger más datos durante el levantamiento del cadáver, por ejemplo respecto a la presunta vía de administración de la droga.

c) Buscar los antecedentes de la persona, tanto los relativos a su posible consumo de cocaína (crónico, esporádico, etc.), como a sus enfermedades.

d) En la persona viva tomar muestras de sangre y de orina y repetir la toma unas horas después, para estimar la dosis y el tiempo transcurrido desde la administración de droga.

e) En el cadáver, tomar muestras de sangre y de orina y también del cerebro y de la bilis. Estos últimos complementan los resultados anteriores y ayudan a estimar la cantidad tomada y el intervalo transcurrido desde entonces. 


\section{BIBLIOGRAFÍA:}

I. US Department of Health and Human Services, Substance Abuse and Mental Health Services Administration. National household survey on drug abuse series: H-IO. Summary of findings from the 1988 national household survey on drug abuse. DHHS publ. no. (SMA) 99 - 3328. Washington DC:USHHS; 1999.

2. Marzuk PM, Tardiff K, , eon AC, Hirsch CS, Stajic M, Portera , , Hartwell N, Iqbal MI. Fatal injuries after cocaine use as a leading cause of death among young adults in New York City. N Engl J Med 1995 Jun 29; 332: 1753-7.

3. Hanzlick R, Gowitt GT. Cocaine metabolite detection in homicide victims. JAMA 199I Feb 13; 265: 760-I.

4. Marzuk PM, Tardiff K, Smyth D, Stajic M, , eon AC. Cocaine use, risk taking, and fatal Russian roulette. JAMA 1992 May 20; 267: 2635-7.

5. Carmen del Rio M, Gomez J, Sancho M, Alvarez FJ. Alcohol, illicit drugs and medicinal drugs in fatally injured drivers in Spain between 199I and 2000. Forensic Sci Int 2002 Jun 25; I27: 63-70.

6. Ellenhorn MJ, Barceloux DG. Medical toxicology. Elsevier, New York, 1988.

7. , adrón J, Moya V. Toxicología médica. Interamericana/McgrawHill, Madrid, 1995.

8. Evans SM, Cone EJ, Henningfield JE. Arterial and venous cocaine plasma concentrations in humans: relationship to route of administration, cardiovascular effects and subjective effects. J Pharmacol Exp Ther 1996 Dec; 279:1345-56.

9. Jenkins AJ, Keenan RM, Henningfield JE, Cone EJ. Correlation between pharmacological effects and plasma cocaine concentrations after smoked administration. J Anal Toxicol 2002 0ct; 26: 382-92.

10. Halpern JH, Sholar MB, Glowacki J, Mello NK, Mendelson JH, Siegel AJ. Diminished interleu-kin6 response to proinflammatory challenge in men and women after intravenous cocaine administration. J Clin Endocrinol Metab 2003 Mar; 88: II88-93.

II. Jufer RA, Walsh S, Cone EJ. Cocaine and metabolite concentrations in plasma during repeated oral administration: development of a human laboratory model of chronic cocaine use. J Anal Toxicol 1998 0ct; 22: 435-44.

12. Isenschmid DS, Fischman MW, Foltin RW, Caplan YH. Concentration of cocaine and metabolites in plasma of humans following intravenous administration and smoking of cocaine. J Anal Toxicol 1992 Sep-0ct; 16: 3II-4.

13. Chow MJ, Ambre JJ, Ruo TI, Atkinson AJ Jr, Bowsher DJ, Fischman MW. Kinetics of cocaine distribution, elimination, and chronotropic effects. Clin Pharmacol Ther 1985 Sep; 38: 318-24.

14. Perez-Reyes M, Jeffcoat AR, Myers M, Sihler K, Cook CE. Comparison in humans of the po-tency and pharmacokinetics of intravenously injected cocaethylene and cocaine. Psychopharmacology (Berl). 1994 Dec; II6: 428-32.

15. Jeffcoat AR, Perez-Reyes M, Hill JM, Sadler BM, Cook CE. Cocaine disposition in humans after intravenous injection, nasal insufflation (snorting), or smoking. Drug Metab Dispos 1989 Mar-Apr; 17: 153-9.
16. Jufer RA, Wstadik A, Walsh S, , evine BS, Cone EJ. Elimination of cocaine and metabolites in plasma, saliva, and urine following repeated oral administration to human volunteers. J Anal Toxicol 2000 0ct; 24: 467-77.

17. Warner A, Norman AB. Mechanisms of cocaine hydrolysis and metabolism in vitro and in vivo: a clarification. Ther Drug Monit 2000 Jun; 22: 266-70.

18. Moolchan ET, Cone EJ, Wstadik A, Huestis MA, Preston K, Cocaine and metabolite elimination patterns in chronic cocaine users during cessation: plasma and saliva analysis. J Anal Toxicol 2000 0ct; 24: 458-66.

19. Drummer OH, Gerostamoulos J. Postmortem drug analysis analytical and toxicological aspects. Therapeutic Drug Monitoring 2002 Apr; 24:199-209.

20. Cone EJ., egal, workplace, and treatment drug testing with alternate biological matrices on a global scale. Forensic Sci Int 200I Sep I5;12I: 7-15.

2I. Vanbinst R, Koenig J, Di Fazio V, Hassoun A. Bile analysis of drugs in post-mortem cases. Fo-rensic Sci Int 2002 Aug 14; 128: 35-40.

22. Hamilton HE, Wallace JE, Shimek E, Jr, , and P, Harris SC, Christenson JG. Cocaine and benzoylecgonine excretion in humans. J Forensic Sci 1977 Oct; 22: 697-707.

23. Spiehler VR, Reed D. Brain concentrations of cocaine and benzoylecgonine in fatal cases. J Forensic Sci 1985 0ct; 30: 1003-II.

24. Mackey-Bojack S, Kloss J, Apple F. Cocaine, cocaine metabolite, and ethanol concentrations in postmortem blood and vitreous humor. J Anal Toxicol 2000 Jan-Feb; 24: 59-65.

25. McKinney PE, Phillips S, Gomez HF, Brent J, MacIntyre M, Watson WA. Vitreous humor cocaine and metabolite concentrations: do postmortem specimens reflect blood levels at the time of death? J Forensic Sci 1995 Jan; 40:102-7.

26. Williams RH, Maggiore JA, Shah SM, Erickson TB, Negrusz A. Cocaine and its major metabolites in plasma and urine samples from patients in an urban emergency medicine setting. J Anal Toxicol 2000 Oct; 24: 478-8I.

27. Shimomura ET, Hodge GD, Paul BD. Examination of postmortem fluids and tissues for the presence of methylecgonidine, ecgonidine, cocaine, and benzoylecgonine using solidphase extraction and gas chromatographymass spectrometry. Clin Chem 2001 Jun; 47: 1040-7. 28. Jenkins AJ, Goldberger BA. Identification of unique cocaine metabolites and smoking byproducts in postmortem blood and urine specimens. J Forensic Sci 1997 Sep; 42: 824-7.

29. Karch SB, Stephens B, Ho CH. Relating cocaine blood concentra-tions to toxicity an autopsy study of 99 cases. J Forensic Sci 1998 Jan; 43: 4I-5.

30. , ogan BK, Smirnow D, Gullberg RG. , ack of predictable sitedependent dif-ferences and timedependent changes in postmortem concentrations of cocaine, benzoylecgonine, and cocaethylene in humans. J Anal Toxicol 1997 Jan-Feb; 2I: 23-3I.

3I. Hearn W, , Keran EE, Wei HA, Hime G. Site-dependent postmortem changes in blood cocaine concentrations. J Forensic Sci 1991 May; 36: 673-84. 